\title{
Proteomic changes of $\mathrm{CD}^{+} / \mathrm{CD}^{+} /$forkhead box $\mathrm{p3}^{+}$ regulatory $T$ cells in a 30 -day rat model of sepsis survival
}

\author{
YUXIA JIAO, SIQI TAN and JUNYU XIONG
}

\begin{abstract}
Department of Anesthesiology, The Second Hospital of Dalian Medical University, Dalian, Liaoning 116027, P.R. China
\end{abstract}
Received December 1, 2016; Accepted July 20, 2017

DOI: 10.3892/etm.2017.5233

\begin{abstract}
Sepsis is defined as life threatening organ dysfunction arising from a dysregulated host response to infection. The outcomes of sepsis include early mortality, delayed mortality and recovery, and depend on the inflammatory response. Previous studies have demonstrated that regulatory $\mathrm{T}$ cells (Tregs) are important in determining the outcome of sepsis, as their suppressive function serves a role in maintaining immune homeostasis. However, Treg-mediated immunosuppression during the course of sepsis remains unclear and little is known about the survival of patients following diagnosis. Studying the survivors of sepsis may explain the mechanisms of natural recovery. Therefore, a 30 -day rat model of sepsis survival was established in the current study. Cluster of differentiation $\mathrm{CD} 4^{+} / \mathrm{CD} 25^{+} /$forkhead box $\mathrm{p}^{+}$Tregs were isolated from the blood and spleens of rats undergoing cecal ligation and puncture or sham surgery, using flow cytometry. Proteomic analysis was performed using nano high-performance liquid chromatography-mass spectrometry. Several different biological pathways associated with uncommon differentially-expressed proteins were identified in the blood and spleen survivor and sham groups. Extracellular-regulated kinase/mitogen-activated protein kinase, as well as integrin and actin cytoskeletal pathway elements, including Ras-related protein $1 \mathrm{~b}$, talin 1 and filamin A, were associated with Tregs in the blood. Pathway elements associated with cell cycle regulators in the B-cell translocation gene family of proteins, tumor necrosis factor receptor superfamily member 4, Hippo signaling, P70-S6 kinase 1, phosphatidylinositol 3-kinase/protein kinase B signaling and 1,25-dihydroxyvitamin D3 biosynthesis were associated with Tregs from the spleen including phosphatase $2 \mathrm{~A}$ activator regulatory factor 4 , histone arginine methyltransferase, CD4, major histocompatibility complex class I antigens, 14-3-3
\end{abstract}

Correspondence to: Professor Junyu Xiong, Department of Anesthesiology, The Second Hospital of Dalian Medical University, 467 Zhongshan Road, Dalian, Liaoning 116027, P.R. China

E-mail: jyxiong0639@163.com

Key words: sepsis, forkhead box $\mathrm{p} 3$, regulatory $\mathrm{T}$ cells, recover, survival model, proteome, biological pathways, blood, spleen protein $\theta$ and nicotinamide adenine dinucleotide phosphate cytochrome $\mathrm{P} 450$ reductase. These results explain the mechanism by which Tregs naturally recover and indicates that Tregs in the blood and spleen vary. Differentially-expressed proteins serving a role in these pathways provide additional insight for the identification of new targets for the diagnosis and treatment of sepsis.

\section{Introduction}

Sepsis is defined as life-threatening organ dysfunction arising from a dysregulated host response to infection. Septic shock is a severe form of sepsis in which circulatory, cellular and metabolic abnormalities increase the risk of mortality more than sepsis alone (1). Sepsis is the primary cause of mortality among critically ill patients in intensive care units (2) and while its incidence continues to increase, the understanding of sepsis, as well as its diagnosis and treatment, remain limited. However, it has been suggested that immunosuppression serves an important role in sepsis (3).

Hotchkiss et al $(4,5)$ demonstrated that early mortality occurs due to an uncontrollable inflammatory cytokine storm, but if pro-inflammatory and anti-inflammatory responses are rebalanced, the prognosis of patients improves, leading to successful recovery. However, if innate and adaptive immune functions are damaged, delayed mortality occurs due to persistent immunosuppression and recurrent infections. The reasons for the different outcomes of sepsis remain unknown. Previous studies have focused on the cause of mortality in patients with sepsis and few experiments have assessed those who survived (2-5). Studying the survivors of sepsis may explain the varying outcomes and mechanisms of recovery. Furthermore, establishing natural causes of sepsis may identify the necessity for intervention in patients with sepsis. Thus, investigations into the immune response that occurs during the recovery stage of sepsis are required.

In the current study, a stable and reliable 30-day sepsis survival model was established in rats. The quality and quantity of regulatory $\mathrm{T}$ cells (Tregs) were observed dynamically to reflect early, delayed and recovery immune status to improve understanding of the differences in immune responses during septic periods. Tregs are specialized immune cells that serve important roles in the maintenance of immune homeostasis (6). Tregs may contribute to immunosuppressive conditions during the course of sepsis, however, their relevance during early, 
delayed and recovery stages of the disease remain unclear and the results of previous studies are contradictory (7-10).

The proteomes of Tregs from the blood and spleens of rats that survived sepsis were obtained using nano high-performance liquid chromatography-mass spectrometry (nano HPLC-MS/MS). It was proposed that differentially-expressed proteins and their associated signaling pathways may explain the mechanisms of recovery from sepsis. Differential protein expression was detected in Tregs from the blood and spleens of rats that survived sepsis and those who underwent sham surgery and this tissue specificity may be associated with the pathogenesis of sepsis. The current study identified the proteins involved in sepsis and may provide novel approaches for the clinical monitoring and treatment of patients with sepsis.

\section{Materials and methods}

Animal model. A total of 110 male Sprague-Dawley rats (weight, 300-350 g; age, 10 weeks) were purchased from Dalian Medical University (Dalian, China) for experiments. Rats were divided into the sham group $(n=42)$ and sepsis group ( $n=68$ ). Rats were housed in standard conditions (room temperature $22^{\circ} \mathrm{C}$; humidity 50-65\%, 12-h light/dark cycle) and had free access to food and water. Rats were allowed to acclimate for $\geq 7$ days before experiments began. All surgical procedures were performed using a small-animal anesthesia machine (Raymain Instrument, Co., Ltd., Shanghai, China) with 3\% sevoflurane (Abbott Laboratories, Lake Bluff, IL, USA). PE-50 catheters (inner diameter, $0.58 \mathrm{~mm}$; outer diameter, $0.96 \mathrm{~mm}$; length, $8 \mathrm{~cm}$; Smiths Medical UK, Kent, UK) were inserted into the right external jugular vein and carotid artery of the rats. Sepsis was induced via cecal ligation and puncture (CLP) $24 \mathrm{~h}$ following insertion of the catheter. The rats were anesthetized and the abdominal cavity was opened with a $2 \mathrm{~cm}$ midline incision. In the sepsis group, the cecum was exposed and ligated $2 \mathrm{~cm}$ from the blind end and below the ileocecal valve, and subsequently punctured 3 times using a 21-gauge triangular needle. A total volume of $0.2 \mathrm{ml}$ fecal material was squeezed from the cecum into the peritoneum. The sham group underwent laparotomy alone, without surgical manipulation of the cecum. A $2 \mathrm{ml}$ volume of $0.9 \%$ normal saline was injected intraperitoneally. The abdomen was slowly manipulated so that the contents were diffused and the intestines were arranged naturally. Following the suturing of the abdominal cavity, $4 \mathrm{mg} / \mathrm{kg} 0.125 \%$ bupivacaine (Shandong Hualu Pharmaceutical Co., Ltd., Laocheng, China) was applied around the incision site for postoperative analgesia in sepsis and sham rats. Following recovery from anesthesia, fluid resuscitation consisting of a 1:1 solution of $6 \%$ hetastarch (Eloheas, Fresenius Kabi Asia-Pacific, Ltd., Wanchai, Hong Kong) and 5\% glucose was administered through the catheters in the right external jugular vein. A total of $20 \mathrm{ml} / \mathrm{kg}$ liquid was administered every $12 \mathrm{~h}$ on the first and second days, which was then halved until the rats resumed eating (11). All experimental animal protocols were approved by the Animal Care and Use Committee of Dalian Medical University.

Sepsis evaluation. All rats were monitored for 30 days. The body weight of the rats was recorded at the same time each day up until mortality. Rats were scored as mildly, moderately or severely affected with regard to appearance, alertness and blood pressure, $24 \mathrm{~h}$ following CLP (Table I). Each rat had to exhibit at least two characteristics in the appearance and alertness categories to obtain a score for that particular category. Blood pressure readings were recorded when the line was clear and the trace was stable for $10 \mathrm{~min}$ (12). Rats were anatomized following mortality or on day 30 when the remaining rats were sacrificed in the survival and sham groups.

Cytokine analysis. Blood samples $(1 \mathrm{ml})$ were collected on days $0,1,7,14,22$ and 30 from 3 rats per group at each time-point and stored in EDTA in anticoagulation tubes on ice. Plasma was separated and stored at $-80^{\circ} \mathrm{C}$ prior to analysis. Lymphocytes were isolated and homogenized in $1 \mathrm{ml}$ 1X PBS and stored overnight at $-20^{\circ} \mathrm{C}$. Three freeze-thaw cycles were performed to break down cell membranes and the resulting homogenates were centrifuged for $5 \mathrm{~min}$ at $5,000 \mathrm{x} \mathrm{g}$ at $2-8^{\circ} \mathrm{C}$. The supernatant was then removed and stored at $-80^{\circ} \mathrm{C}$. Levels of interleukin (IL)-10, transforming growth factor $\beta 1$ (TGF- $\beta 1$ ) and forkhead box p3 (Foxp3) were analyzed using ELISA kits: Rat IL-10 (cat no. CSB-E04595r), rat TGF- $\beta 1$ (cat no. CSB-E04727r) and rat FoxP3 (cat no. CSB-E15075r) (all from Cusabio Biotech, Co., Ltd., Wuhan, China). The minimum detectable levels of these proteins are typically $<0.78,1.56$ and $7.81 \mathrm{pg} / \mathrm{ml}$, respectively, according to the manufacturer.

Treg analysis. A total of three rats per group at each time-point were sacrificed on days $0,1,7,14,22$ and 30. Lymphocytes were isolated from the blood and spleen samples (rat peripheral blood/spleen lymphocytes isolation kit, LTS1083/1083PK; Tianjin Haoyang Biological Manufacture Co., Ltd., Tianjin, China) to conduct staining and flow cytometry. Anti-rat CD4-fluorescein isothiocyanate [cat no. 11-0040, 1:200, anti-rat CD25-phycoerythrin (PE)] (cat no. 12-0390, 1:150) or their isotype control antibodies (cat no. 11-4724, 1:200; cat no. 12-4724, 1:150) were added to $100 \mu \mathrm{l}$ cell suspension $\left(10^{6}\right.$ cells) in the dark for $30 \mathrm{~min}$ at $4^{\circ} \mathrm{C}$. Cells were then washed twice with staining buffer (cat no. 00-4222). Following fixation/permeabilization (cat no. 00-5523) in the dark for $30 \mathrm{~min}$ at $4^{\circ} \mathrm{C}$, anti-mouse/rat Foxp3-PE-cyanine5 (cat no. 15-5773, $1: 10$ ) or its isotype control antibody (cat no. 15-4321, 1:10) were added and incubated in the dark for $30 \mathrm{~min}$ at $4^{\circ} \mathrm{C}$. Cells were then washed twice with permeabilization buffer (cat no. 00-5523) and the population of $\mathrm{CD} 4^{+} \mathrm{CD} 25^{+} \mathrm{T}$ cells and $\mathrm{CD} 4{ }^{+} \mathrm{CD} 25^{+} \mathrm{Foxp}^{+}$Tregs was analyzed using a flow cytometer with FACSDiva 7.0 software (BD Biosciences, Franklin Lakes, NJ, USA). All antibodies, isotype control antibodies and buffers were purchased from eBioscience; Thermo Fisher Scientific, Inc. (Waltham, MA, USA).

Proteomic analysis. A total of 8 rats from the survival group and 8 rats from the sham group were sacrificed on day 30 and $\mathrm{CD}^{+}{ }^{+} \mathrm{CD} 25^{+} \mathrm{Foxp} 3^{+}$Tregs were isolated from the blood and spleen samples using a flow cytometer. Proteins were denatured with a $\mathrm{pH} 7.4$ extraction buffer consisting of $8 \mathrm{M}$ guanidine hydrochloride, $2 \mathrm{M}$ urea and a $2 \%$ protease inhibitor cocktail. Cells were disrupted using ultra sonication with 10 cycles of $5 \mathrm{sec}$ work-time and $10 \mathrm{sec}$ recovery $(\mathrm{kHz}, \mathrm{w})$ on 
Table I. Sepsis scoring rubric.

\begin{tabular}{|c|c|c|c|}
\hline Variable & Mild & Moderate & Severe \\
\hline \multirow[t]{3}{*}{ Appearance } & Hunched & Hunched & Marked piloerection \\
\hline & Piloerection & Marked piloerection & Markedly bloated abdomen \\
\hline & No bloating & $\begin{array}{l}\text { Bloated abdomen } \\
\text { Sunken eyes }\end{array}$ & Conjunctival injection \\
\hline \multirow[t]{3}{*}{ Alertness } & Alert & $\begin{array}{l}\text { Depressed level of } \\
\text { alertness }\end{array}$ & $\begin{array}{l}\text { Markedly (or absent) } \\
\text { depressed level of alertness }\end{array}$ \\
\hline & $\begin{array}{l}\text { Occasional interest in } \\
\text { environment }\end{array}$ & $\begin{array}{l}\text { Little interest in } \\
\text { environment }\end{array}$ & No interest in environment \\
\hline & Moves freely & Moves with difficulty & No movement \\
\hline $\begin{array}{l}\text { Mean blood pressure } \\
(\mathrm{mmHg})\end{array}$ & $>90$ & $75-90$ & $>75$ \\
\hline
\end{tabular}

ice. Following incubation for $2 \mathrm{~h}$ at $4^{\circ} \mathrm{C}$, the supernatant was collected using centrifugation at $35,000 \mathrm{x}$ g for $45 \mathrm{~min}$ at $4^{\circ} \mathrm{C}$.

A total of $100 \mu \mathrm{g}$ protein was reduced with $100 \mathrm{mM}$ dithiothreitol for $5 \mathrm{~min}$ at $95^{\circ} \mathrm{C}$ and the supernatant was collected following centrifugation at $20,000 \times \mathrm{g}$ for $30 \mathrm{~min}$ at room temperature. Proteins were alkylated with $50 \mathrm{mM}$ iodoacetamide (Sigma-Aldrich; Merck KGaA, Darmstadt, Germany) for $20 \mathrm{~min}$ in the dark at room temperature and then transferred to an ultrafiltration membrane $(10 \mathrm{kDa}$; Sartorius AG, Göttingen, Germany) by centrifugation at 14,000 x $\mathrm{g}$ for $20 \mathrm{~min}$ at room temperature. The membrane was incubated with $2 \mu \mathrm{g}$ trypsin for $12 \mathrm{~h}$ in a water bath at $37^{\circ} \mathrm{C}$ and then washed with $100 \mu 150 \mathrm{mM}$ phosphate buffer $(\mathrm{pH} \mathrm{8.5)}$ and centrifuged three times at $14,000 \times \mathrm{g}$ for $20 \mathrm{~min}$ at room temperature.

Peptide samples obtained from Tregs in the survival-group blood and sham-group spleens were labeled with $16 \mu \mathrm{l}$ $4 \%$ formaldehyde and classified as light label (L), whereas peptide samples from Tregs in the sham-group blood and survival-group spleens were labeled with $16 \mu 14 \%$ deuterated formaldehyde and classified as heavy label (H). Samples were mixed by vortexing at 1,000 rpm for $3 \mathrm{~min}$ and $16 \mu 1$ sodium cyanoborohydride was added. Following $1 \mathrm{~h}$ agitation at $37^{\circ} \mathrm{C}$, the reaction was attenuated with $1 \mu 125 \%$ ammonia. $\mathrm{pH}$ was tested using $1 \mu \mathrm{l}$ formic acid and the results indicated that the sample was acidic. The labeled samples from the survival-group and sham-group blood and spleens were mixed at a 1:1 ratio prior to nano HPLC-MS/MS.

Nano HPLC-MS/MS. Nano HPLC was performed using an UltiMate 3000 Capillary/Nano LC system equipped with an autosampler (Dionex; Thermo Fisher Scientific, Inc.) at room temperature. Peptide separation was performed on a Venusil XBP C18 column (4.6x150 mm, $5 \mu \mathrm{m}, 100 \AA$; Tianjin Bonna-Agela, Tianjin, China) with buffers as follows: i) Mobile phase A consisting of $2 \%$ volume/volume (v/v) acetonitrile with $0.1 \%(\mathrm{v} / \mathrm{v})$ formic acid and ii) mobile phase $\mathrm{B}$ consisting of $98 \%(\mathrm{v} / \mathrm{v})$ acetonitrile with $1.0 \%(\mathrm{v} / \mathrm{v})$ formic acid. Following pretreatment of the analytical column $(150 \mathrm{~mm}$, $75 \mu \mathrm{m}$ inside diameter) with $98 \%$ mobile phase $\mathrm{A}, 1 \mu \mathrm{g}$ labeled samples were loaded onto a trap column using the autosampler at a flow rate of $7 \mu \mathrm{l} / \mathrm{min}$. The gradients for separation were as follows: $15-100 \mathrm{~min}, 6-22 \%$ mobile phase B; 100-120 min, 22-35\% mobile phase B; $120-130$ min, $35-8 \%$ mobile phase B and $130-140 \mathrm{~min}, 80 \%$ mobile phase $\mathrm{B}$.

The LC eluent was analyzed with positive ion nanoflow electrospray using a $\mathrm{Q}$ Exactive $^{\mathrm{TM}}$ Benchtop Quadrupole-Orbitrap ${ }^{\mathrm{TM}}$ mass spectrometer (Thermo Fisher Scientific, Inc.). The parameters of the first stage mass spectrum were as follows: The spray voltage of the capillary was $1.6 \mathrm{kV}$; the MS mass scan range was $300-1,800 \mathrm{~m} / \mathrm{sec}$ with a resolution of 70,000; the ion automatic gain control (AGC) was set to $1 \mathrm{e} 6$ and the maximum time of ion injection was $50 \mathrm{~ms}$. The data-dependent acquisition parameters of the second stage were as follows: The strongest ten ions of which the MS spectrum intensity was $>1 \mathrm{e} 4$ were selected in a dynamic exclusion; the collision energy was set to $28 \%$; the resolution was 17,500; the ion AGC was 1e4; the maximum time of ion injection was $100 \mathrm{~ms}$ and selected ions were isolated with $2 \mathrm{~m} / \mathrm{s}$. MS data were collected and recorded using Xcalibur software (version 3.0.63; Thermo Fisher Scientific, Inc.). Nano HPLC-MS/MS analysis was performed on each sample in triplicate.

Data analysis. The results were analyzed with MaxQuant software (version 1.5.2.8; Max Planck Institute of Biochemistry, Munich, Germany) using the SP Rat database (www.uniprot.org). Cysteine residues were given a fixed modification of +57.0215 Da. The N-terminus Lys was set to +28 Da and +32 Da dimethylated variable modifications. Peptide retrieval was performed using trypsin complete digestion and tolerance for up to two leaky cut sites. The parent ion mass tolerance had a deviation of $20 \mathrm{ppm}$ and the mass fragment ion tolerance had a deviation of $0.5 \mathrm{Da}$. The control of search results on the level of protein and peptide segments had a false positive rate of $<1 \%$. Proteins were identified using Uniprot Knowledgebase (www.uniprot.org) software for cellular localization and functional analysis of proteins. Proteins with $\geq 2$ spectral counts in a technical replicate were considered for further analysis. Peptides with a relative standard deviation of $<50 \%$ of protein ratios were selected. Proteins with average ratios of $<0.67$ or $>1.5$ were differentially expressed 
A

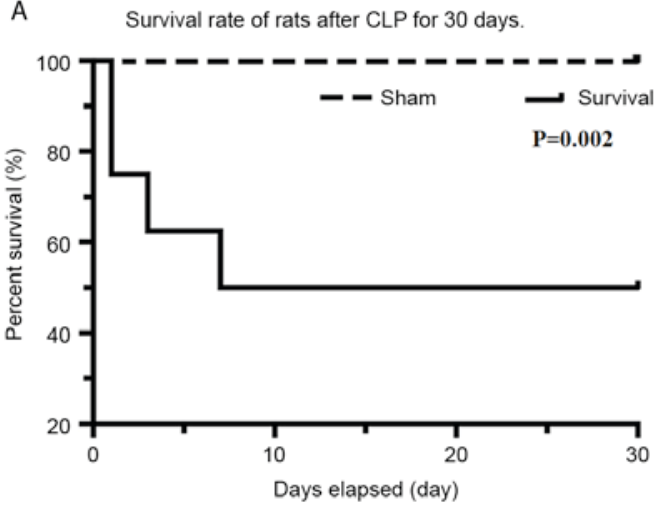

C

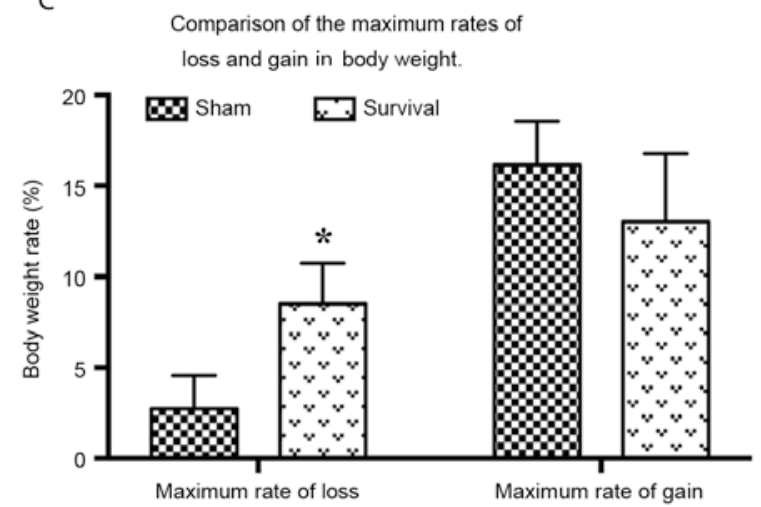

B

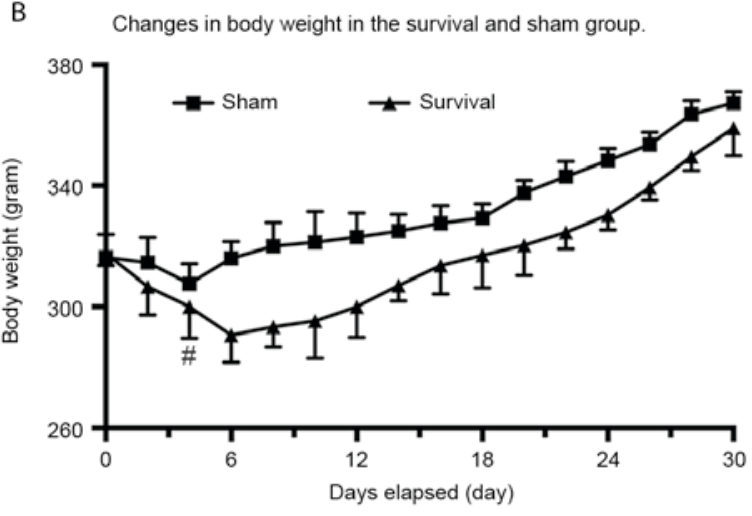

D

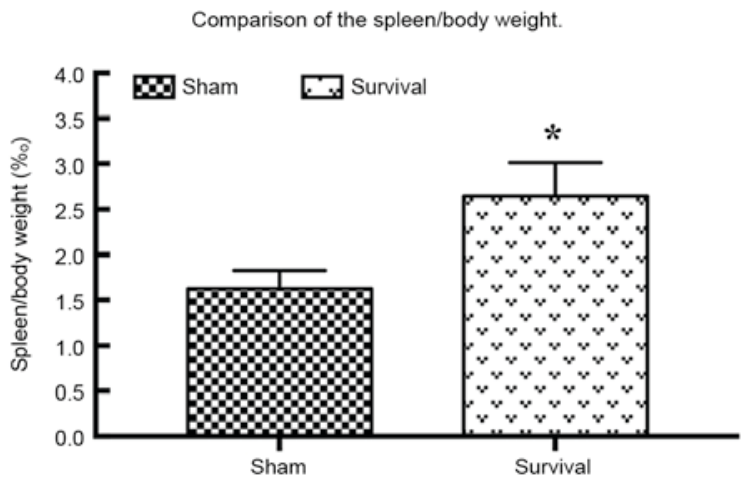

Figure 1. Evaluation of survival model. (A) Survival rate of rats 30 days following CLP ( $n=16)$. (B) Changes in body weight in the survival and sham groups $(n=8)$. (C) Comparison of the maximum rates of loss and gain of body weight in the survival and sham groups. (D) Comparison of the spleen and body weight in the survival and sham groups $(\mathrm{n}=8)$. Data are expressed as the mean \pm standard error of the mean. ${ }^{~} \mathrm{P}<0.05$. CLP, cecal ligation and puncture. ${ }^{~} \mathrm{P}<0.05$ vs. Day $0 ;{ }^{*} \mathrm{P}<0.05$ vs. Sham group.

in a significant manner. The identified proteins were analyzed using Ingenuity Pathway Analysis (IPA; www.ingenuity.com).

Statistical analysis. Data are expressed as the mean \pm standard error of the mean. The differences among groups over time were analyzed using a repeated measures analysis of variance followed by a post hoc Fisher's least significant difference test using SPSS 17.0 (SPSS, Inc., Chicago, IL, USA). P<0.05 was determined to indicate a statistically significant difference. The survival curves were generated using the Kaplan-Meier method and compared using the log-rank Mantel-Cox test.

\section{Results}

Evaluation of the survival model. CLP caused sepsis with a medium-severe severity, as assessed by appearance, alertness and blood pressure defined on the first day following CLP. The survival rate of rats following CLP was $75 \%$ on day $1,62.5 \%$ on day 3 and $50 \%$ on day 7 compared with $100 \%$ in the sham group (Fig. 1A). There was a significant decrease $(\mathrm{P}=0.017)$ in weight in the survival group over the 4 days following CLP, with the minimum observed on day 6 (Fig. 1B). The highest body weight loss compared with original weight was $8.51 \pm 2.23 \%$ and the difference between weight loss observed in the two groups was significant $(\mathrm{P}=0.026$; Fig. $1 \mathrm{C})$. In the survival group, the anatomy of the abdomen exhibited adhesions in the abdominal cavity and the omentum had lost shape and gloss, and trended towards ligation of the intestine. No encapsulated abscesses were observed in the survival group. The ratio of spleen weight to total body weight was $2.64 \pm 0.37 \%$ in the survival group and $1.63 \pm 0.20 \%$ in the sham group. The difference between the two groups was significant ( $\mathrm{P}=0.032$; Fig. 1D).

Immune performance of $C D 4^{+} C D 25^{+} F_{0 x p} 3^{+}$Tregs. The concentration of IL-10 on day 1 was significantly increased in the survival $(\mathrm{P}=0.007)$ and succumbed $(\mathrm{P}=0.011)$ groups compared with the sham group. The difference in IL-10 concentration between animals that survived and those that succumbed was also significant $(\mathrm{P}=0.004)$ however, IL-10 levels fell to baseline on day 7 (Fig. 2A). The concentration of TGF- $\beta 1$ was significantly increased in the survival $(\mathrm{P}=0.004)$ and succumbed groups on day $1(\mathrm{P}=0.046)$ compared with the sham group. However, TGF- $\beta 1$ levels fell to baseline on day 7 (Fig. 2B). The concentration of Foxp3 was significantly increased up until day 30 in the survival group $(\mathrm{P}=0.024$, $0.006,0.003,0.002$ and 0.004 at day 1, 7, 1422 and 30, respectively) compared with the sham group (Fig. 2C).

The proportion of $\mathrm{CD} 4^{+} \mathrm{CD} 25^{-} \mathrm{T}$ cells, representing lymphocytes in the blood, began to decrease 1 day following CLP and was significantly reduced on days $7(\mathrm{P}=0.026)$ and $14(\mathrm{P}=0.030)$ in the survival group compared with the sham group (Fig. 2D). These values increased 22 days following 


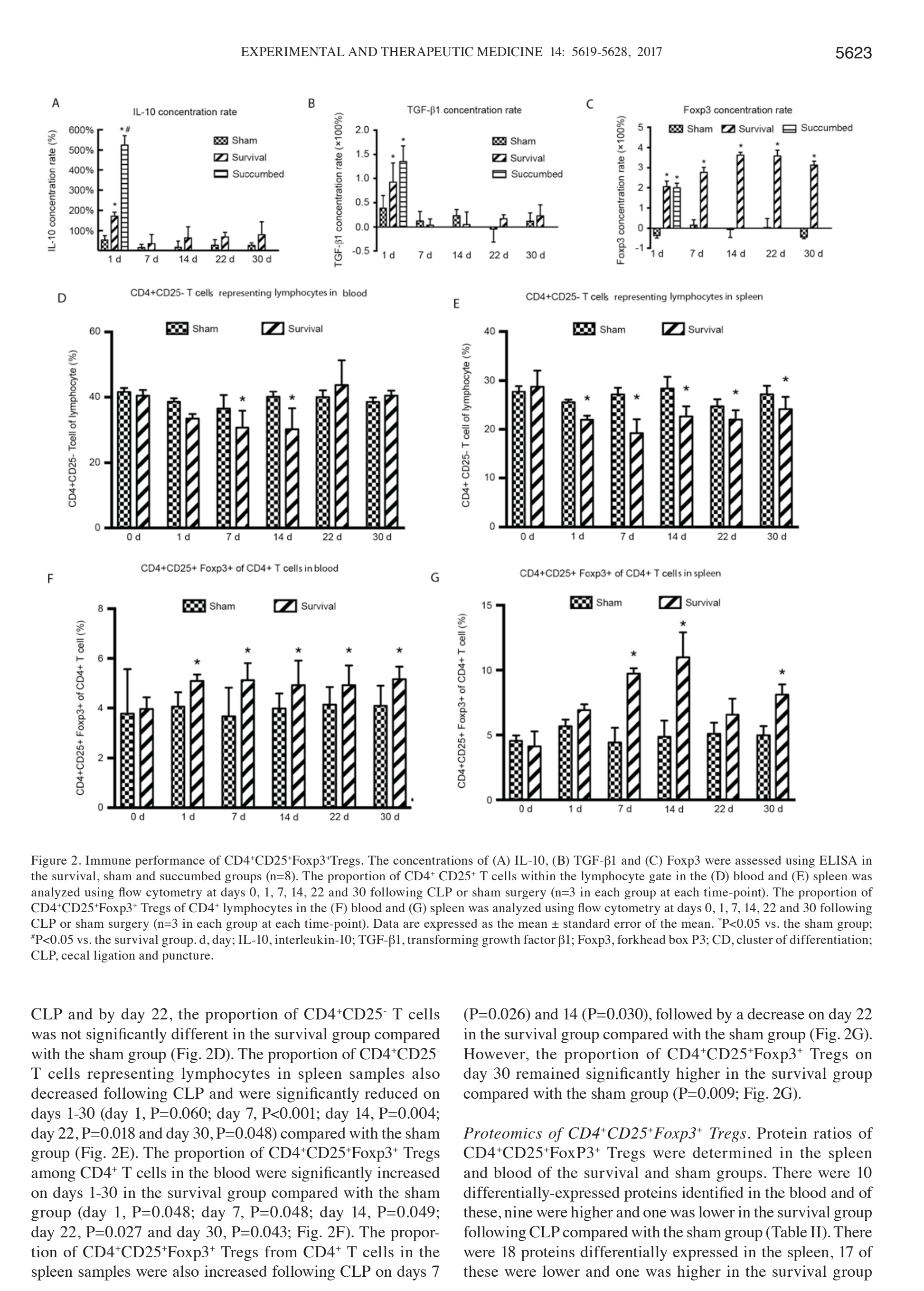


Table II. Proteins identified in blood $\mathrm{CD} 4^{+} \mathrm{CD} 25^{+} \mathrm{FoxP} 3^{+}$Tregs, which are differently expressed between survival and sham groups.

\begin{tabular}{|c|c|c|c|c|}
\hline Entry name & Gene & Protein & $\begin{array}{c}\text { H/L Ratio } \\
\text { (mean } \pm \text { standard } \\
\text { deviation) }\end{array}$ & $\begin{array}{c}\text { Ratio of } \\
\text { standard } \\
\text { deviation }\end{array}$ \\
\hline Q62636 & RAP1B & Ras-related protein Rap-1b & $1.52 \pm 0.04$ & 0.03 \\
\hline C0JPT7 & FLNA & Filamin A & $1.53 \pm 0.11$ & 0.07 \\
\hline G3V852 & TLN1 & Talin 1 (isoform CRA_b) & $1.53 \pm 0.60$ & 0.39 \\
\hline B2GVB9 & FERMT3 & Fermitin family homolog 3 & $1.66 \pm 0.13$ & 0.08 \\
\hline P02091 & $\mathrm{HBB}$ & Hemoglobin subunit $\beta-1$ & $1.80 \pm 0.46$ & 0.26 \\
\hline Q566D6 & PSIP1 & PC4 and SFRS1-interacting protein & $1.88 \pm 0.32$ & 0.17 \\
\hline B1H216 & HBZ & Hemoglobin subunit $\zeta$ & $1.88 \pm 0.51$ & 0.27 \\
\hline B2RYQ5 & $\mathrm{ERH}$ & Enhancer of rudimentary homolog & $1.90 \pm 0.61$ & 0.32 \\
\hline Q63011 & $\mathrm{N} / \mathrm{A}$ & Zero $\beta$-globin (Fragment) & $2.22 \pm 0.17$ & 0.08 \\
\hline Q4KLJ1 & SRSF7 & $\begin{array}{l}\text { Serin/arginine rich splicing factor } \\
\text { (isoform CRA_a) }\end{array}$ & $0.54 \pm 0.12$ & 0.22 \\
\hline
\end{tabular}

$\mathrm{n}=8$ in each group. CD, cluster of differentiation; H/L, heavy/light; Tregs, regulatory T-cells. Heavy group, Tregs from the blood of the sham group; Light, Tregs from the blood of the survival group.

Table III. Proteins identified in the spleen $\mathrm{CD} 4^{+} \mathrm{CD} 25^{+} \mathrm{FoxP} 3^{+}$Tregs, which are differentially expressed between survival and sham groups.

\begin{tabular}{llllc}
\hline $\begin{array}{l}\text { Entry } \\
\text { name }\end{array}$ & Gene & \multicolumn{1}{c}{ Protein } & $\begin{array}{c}\text { H/L ratio } \\
\text { (mean } \pm \text { standard } \\
\text { deviation) }\end{array}$ & $\begin{array}{r}\text { Ratio of } \\
\text { standard } \\
\text { deviation }\end{array}$ \\
\hline D3ZK97 & H3F3C & Histone H3.3C & $0.12 \pm 0.04$ & 0.34 \\
F1M2K3 & SUMO2 & Small ubiquitin-related modifier 2 & $0.13 \pm 0.02$ & 0.16 \\
A7LNF8 & RT1.A & RT1 class 1, A1 (Fragment) & $0.14 \pm 0.05$ & 0.37 \\
Q9Z0V5 & PRDX4 & Peroxiredoxin-4 & $0.18 \pm 0.02$ & 0.12 \\
Q3KR55 & U2AF1 & Splicing factor U2AF 35 kDa subunit & $0.20 \pm 0.06$ & 0.31 \\
Q5XII7 & SNRPB & Small nuclear ribnucleoprotein-associated & $0.25 \pm 0.05$ & 0.20 \\
& & proteins B and B' & & 0.10 \\
S5RZM8 & COX2 & Cytochrome coxidase subunit 2 & $0.27 \pm 0.05$ & 0.17 \\
B2RYQ2 & PPP2R4 & Phosphatase 2A activator regulatory factor 4 & $0.27 \pm 0.01$ & 0.03 \\
P68255 & YWHAQ & 14-3-3 protein $\theta$ & $0.34 \pm 0.00$ & 0.13 \\
Q5RKJ9 & RAB10 & Ras-related protein Rab-10 & $0.35 \pm 0.05$ & 0.14 \\
Q6PDV1 & LYZ2 & Lysozyme C-2 & $0.41 \pm 0.03$ & 0.07 \\
P62329 & TMSB4X & Thymosin $\beta$-4 & $0.41 \pm 0.03$ & 0.10 \\
Q63009 & PRMT1 & Protein arginine N-methyltransferase 1 & $0.43 \pm 0.06$ & 0.13 \\
F1LVR0 & IGLON5 & IgLON family member 5 & $0.45 \pm 0.05$ & 0.10 \\
Q6B345 & S100A11 & S100 calcium-binding protein A11 & $0.45 \pm 0.11$ & 0.23 \\
Q5XIP6 & FEN1 & Flap endonuclease 1 & $0.47 \pm 0.01$ & 0.02 \\
P00388 & POR & NADPH-cytochrome P450 reductase & $2.40 \pm 0.30$ & 0.13 \\
P05540 & CD4 & T-cell surface glycoprotein CD4 & \\
\hline
\end{tabular}

$\mathrm{n}=8$ in each group. $\mathrm{CD}$, cluster of differentiation; H/L, heavy/light; Tregs, regulatory T-cells. Heavy group, Tregs from the spleen of the sham group; Light, Tregs from the spleen of the survival group.

compared with the sham group (Table III). It was identified using IPA analysis that 12 biological pathways were associated with the 10 differentially-expressed proteins in the blood
$(\mathrm{P}<0.05$; Fig. 3A). The pathways exhibiting the most significant associations included the extracellular signal-regulated kinase/mitogen-activated protein kinase(ERK/MAPK), as well 
A

A Biological pathways associated with differentially expressed proteins of $\mathrm{CD} 4^{+} \mathrm{CD} 25^{+}$Foxp $3^{+}$Tregs in blood

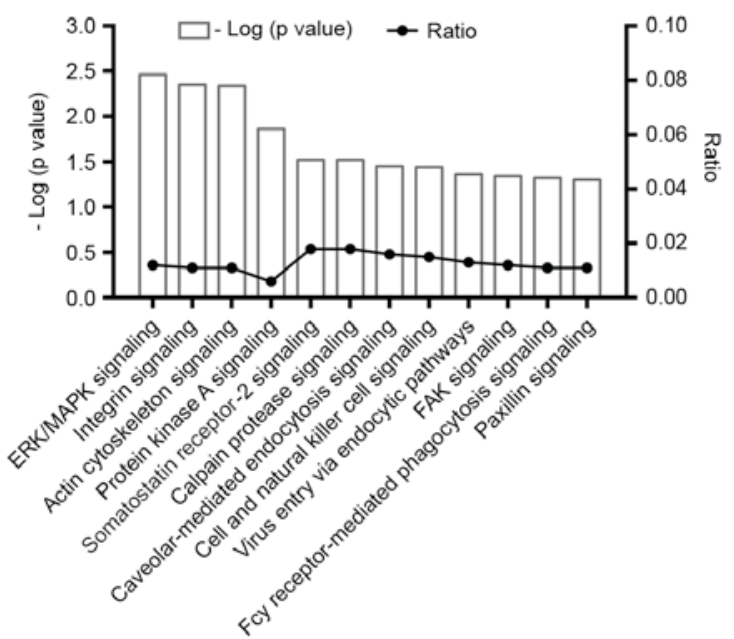

B

Biological pathways associated with differentially expressed proteins of $\mathrm{CD} 4^{+} \mathrm{CD} 25^{+} \mathrm{Foxp} 3^{+}$Tregs in spleen

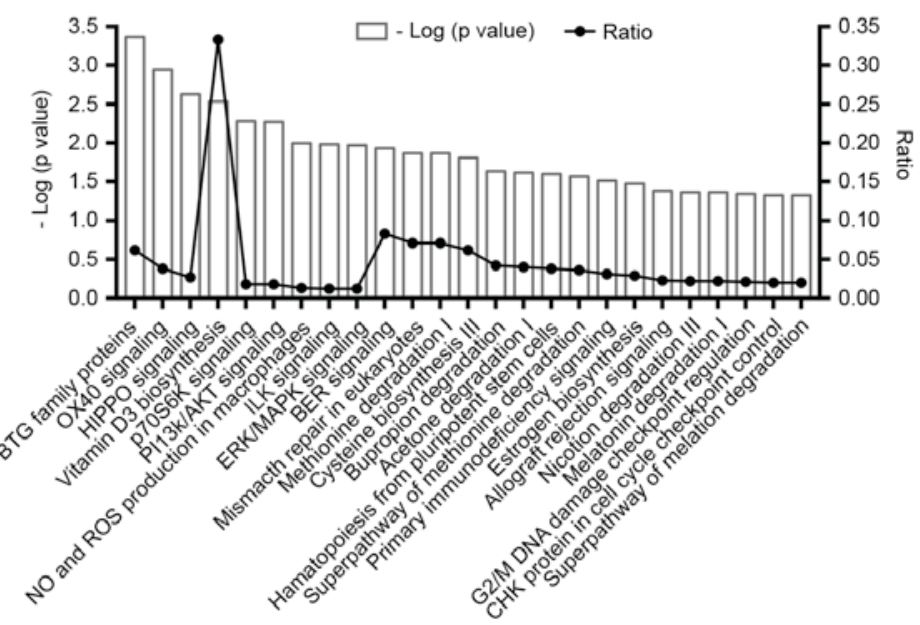

Figure 3. Significant biological pathways associated with differentially expressed proteins of CD4 $4^{+} \mathrm{CD} 25^{+} \mathrm{Foxp} 3^{+}$Tregs in the $(\mathrm{A})$ blood and $(\mathrm{B})$ spleens of the survival group. $\mathrm{P}<0.05=-\log (\mathrm{P}-\mathrm{value})>1.301$. Foxp3, forkhead box P3; CD, cluster of differentiation; ERK/MAPK, extracellular signal-regulated kinase/mitogen-activated protein kinase; FAK, focal adhesion kinase; Fcy, purine cytosine permease; BTG, B-cell translocation gene; p70S6K, P70-S6 kinase 1; PI3K/AKT, phosphatidylinositol 3-kinase/protein kinase B; NO, nitric oxide; ROS, reactive oxygen species; ILK, integrin-linked kinase; BER, base excision repair; CHK, serine/threonine-protein kinase; Treg, regulatory T-cell.

as integrin and actin cytoskeletal signaling pathways (Fig. 3A), in which Ras-related protein $1 \mathrm{~b}$ (Rap-1b), talin-1 (Tln1) and filament protein A (Flna) were associated (Table II). A total of 25 signaling pathways were identified by IPA in the spleen group $(\mathrm{P}<0.05)$. The pathways exhibiting the most significant association with the differentially-expressed proteins included the B-cell translocation gene (BTG) family of proteins that regulate cell cycle pathways, OX40 signaling, Hippo signaling, P70-S6 kinase 1 (P70S6K) signaling, biosynthesis of 1,25-dihydroxyvitamin D3 (VD3) and phosphatidylinositol 3-kinase/protein kinase B (PI3K/AKT) signaling (Fig. 3B). Differential factors generating these results included protein phosphatase 2A activator regulatory subunit 4 (PPP2R4), protein arginine $\mathrm{N}$-methyltransferase 1 (PRMT1), T cell surface glycoprotein CD4 (CD4), RT1.A, 14-3-3 protein $\theta$ (Ywhaq) and nicotinamide adenine dinucleotide phosphate (NADPH)-cytochrome P450 reductase (Table III).

\section{Discussion}

A long-term survival model of sepsis was successfully established in rats using modified CLP procedures, screening for sepsis manifestation and changes in body weight, cytokines and anatomy $(13,14)$ to determine the mechanism by which survivors recover from sepsis without treatment. The current study investigated the immune status and function of Tregs and demonstrated that they are different during the early (first 1-3 days), delayed (7 days later) and recovery (day 30) stages of sepsis (5).

The current study identified 10 differentially-expressed proteins in the blood of the survival/sham groups and 18 differentially-expressed proteins in the spleen of the survival/sham groups. The differentially-expressed proteins and their associated biological pathways differed between Tregs isolated from the blood and spleen.
The ERK/MAPK signaling pathway serves an important role in transducing cellular information about meiosis/mitosis, growth, differentiation and carcinogenesis within a cell. Membrane-bound receptor tyrosine kinases (RTKs) are growth factor receptors, which are the starting point of this pathway. Mitogens, including TGF- $\beta 1$, epidermal growth factor and insulin, activate the ERK signaling pathway. Lipopolysaccharide also stimulates the activation of ERK/MAPK signaling in monocytes/macrophages and endothelial cells (15-17). Phosphorylated RTKs activate Ras, which initiates a kinase cascade, beginning with Raf. This then activates and phosphorylates MAPK, which activates and phosphorylates ERK. This kinase cascade regulates cell growth, differentiation, environmental stress, inflammatory responses and other important cellular physiological/pathological processes (18-20).

The current study demonstrated that the Ras-associated protein Rap-1b serves a role in the ERK/MAPK pathway. Rap- $1 b$ is a member of the Ras superfamily, which is a $22-\mathrm{kDa}$ small GTP-binding protein activated by cAMP-dependent protein kinase. Rap-1b is a key component in the signaling pathway mediated by RTKs (21). It has been demonstrated that Rap-1b phosphorylation serves a role in the biological processes associated with initial cytoskeleton formation and remodeling (21).

RTK activation of Ras and Raf may occur via alternate pathways. For example, integrins, which are cell surface glycoproteins involved in cell-cell and cell-extracellular matrix interactions, activate ERK via a focal adhesion kinase-mediated pathway $(22,23)$. These interactions form the basis of diverse effects including cell migration, anchorage, growth and differentiation (24). The actin cytoskeleton serves an important role in many dynamic processes, including cell motility, axon guidance, cytokinesis and phagocytosis. These cellular remodeling events require precise regulation of actin 
filament assembly/disassembly and organization (24). Integrin signaling is a pathway that controls rearrangement of the actin cytoskeleton (24). It has been suggested that the actin cytoskeleton may regulate receptor signaling, which is particularly important for the B-cell receptor and dysregulated signaling may result in autoimmunity and malignancy (25).

Talin-1 is another differentially-expressed protein, which was the first cytoskeletal protein identified as binding directly to integrin $(26,27)$. Talin serves an important role in the dynamic process of cell adhesion by directly interacting with integrin and the cytoskeleton $(26,27)$. Stem cells with disrupted talin genes cannot form focal adhesions (28). Filamin A was identified in non-muscle cells as the first actin cross-linking protein and acts to stabilize the cytoskeleton, transduce biological signals and also participates in cell dynamics (29-33).

Rap-1b, talin and filamin A are involved in the ERK/MAPK, integrin and actin cytoskeleton signaling pathways, which all interact with each other. Elevated levels of these three proteins suggest that Tregs from the blood continue to proliferate, aggregate and undergo adhesion and phagocytosis 30 days following CLP. Furthermore, Tregs may possess strong immunosuppressive functions. The results of the current study indicated this, as they identified an increased percentage of Tregs using flow cytometry and increased FoxP3 concentration using ELISA.

BTG family members containing a specific BTG domain inhibit cell proliferation (34). BTG regulates cell cycle progression, inhibits proliferation, promotes apoptosis and stimulates differentiation in multiple cell types. The BTG family of proteins inhibit cell proliferation by methylation. PRMT1 is a differentially-expressed protein that serves a key role in this process. The BTG family proteins interact with PRMT1 to increase its methyl-transferase activity (34-36). PRMT1 activity is essential for growth factor-induced cell differentiation, whereas blocking PRMT1 with the Box-C domain of BTG1/2 induces apoptosis $(35,36)$.

OX40 is a $\mathrm{T}$ cell activator that may promote the survival and prolong the immune response of $\mathrm{CD}^{+} \mathrm{T}$ cells at sites of inflammation. The co-stimulatory OX40 ligand is a member of the tumor necrosis factor super family and possesses a transmembrane segment, as well as an extracellular region (37). OX40 signaling synergizes with Toll-like receptors to inhibit Tregs (37). Vu et al (38) demonstrated that co-stimulation with OX40 inhibits Tregs.

The RT1 complex is a rat major histocompatibility (MHC) antigen containing a class of highly polymorphic gene clusters. Among them, the most typical are the MHC class I (RT1.A) and class II (RT1.B) antigens. RT1.A is expressed at a high density on lymphocytes $(39,40)$. Tregs from the spleen of sepsis survivors expressed CD4 and RT1.A, two proteins involved in the OX40 signaling pathway, suggesting it may be a key negative regulator of sepsis.

The Hippo signaling pathway controls tissue growth and homeostasis and serves an essential role in innate immunity (41-43). Transcriptional co-activator YAP1 (YAP) is a major downstream effector of this pathway that translocates into the nucleus upon dephosphorylation, whereby it induces expression of the genes that promote cell proliferation and inhibit apoptosis $(44,45)$. It has been demonstrated that YAP is a functional link between the Hippo signaling pathway and
PI3K-mammalian target of rapamycin (mTOR), providing a molecular basis for the coordination of the two pathways (46). mTOR is a serine/threonine kinase that regulates multiple cellular functions in response to amino acids and grow th factors. mTOR regulates mRNA translation and cell cycle progression via phosphorylation of p70S6K, a serine/threonine kinase that phosphorylates the ribosomal S6 subunit, a component of the $40 \mathrm{~S}$ subunit of eukaryotic ribosomes $(47,48)$. Additionally, mTOR is a key factor in PI3K/AKT signaling and activates downstream substrates to regulate cell growth $(47,48)$. PI3Ks are a family of lipid kinases, which are inositol lipid products serving an important role in the signal transduction pathways of cytokines, growth factors and other extracellular matrix proteins (49). AKT inhibits apoptosis by phosphorylating the Bad component of the Bad/B-cell lymphoma-extra large (Bcl-XL) complex, thus facilitating cell survival. Furthermore, AKT activates $\mathrm{I} \kappa \mathrm{B}$ kinase, leading to nuclear factor $\kappa \mathrm{B}$ activation and enhancing cell survival (50). AKT is involved in numerous cellular processes, including energy storage, cell cycle progression, protein synthesis and angiogenesis $(49,50)$.

Protein phosphatase 2A ( $\mathrm{PP} 2 \mathrm{~A})$ is a ubiquitous enzyme that promotes basal phosphatase activity to serve as a negative regulator of cell growth and division $(51,52)$. The 14-3-3 family of highly conserved proteins bind to phosphorylated serine or threonine peptides to regulate cell growth and metabolic processes (53). The present study demonstrated that Ppp2r4 and Ywhaq were differentially expressed and this differential expression was associated with upregulation of the Hippo, P70S6K and PI3K/AKT signaling pathways to balance cell apoptosis and proliferation.

VD3 is the biologically active form of vitamin D and is considered to be a hormone. While the classic role of vitamin $\mathrm{D}$ is the regulation of calcium and metabolism in bone, it also exhibits immunomodulatory effects by targeting various immune cells, including monocytes, macrophages, dendritic cells and T- and B-lymphocytes $(54,55)$. As well as being targets, immune cells express vitamin D-activating enzymes, allowing local conversion of inactive vitamin $\mathrm{D}$ into VD3 within the immune system (56). The identity of the enzyme that catalyzes the hydroxylation of VD3 at carbon 25 remains unclear. However, it has been suggested that cytochrome $\mathrm{P} 450$ (CYP) is the rate-limiting enzyme in this pathway and hydroxylates VD3 to calcidiol (57). Cytochrome P450 oxidoreductase is a differentially-expressed protein and is the essential electron donor for all CYP enzymes and is responsible for CYP activation $(58,59)$. In Tregs in the spleen, antiproliferative pathways, including cell cycle regulation by BTG family proteins, coexist with proliferative pathways, such as VD3 biosynthesis signaling. This indicates that Tregs in the spleen may be rebalanced 30 days following CLP. Flow cytometry demonstrated that the percentage of splenic Tregs recovered to baseline levels by day 30 .

In conclusion, the present study investigated the quality and quantity of Tregs in a long-term survival model of sepsis. Surviving rats that appeared to have recovered from sepsis exhibited a higher level of Tregs based on the results of cytokine analysis 30 days following CLP. The changes observed in Tregs harvested from the blood and spleen varied. Several biological pathways were identified using a proteomics approach. Uncommon proteins differentially 
expressed between the blood and spleen resulted in different pathways being highly expressed. The ERK/MAPK, integrin and actin cytoskeleton signaling pathways were upregulated in the blood, whereas signaling pathways associated with BTG family proteins (cell cycles regulators), OX40, Hippo, P70S6K, $\mathrm{PI} 3 \mathrm{~K} / \mathrm{AKT}$ and VD3 biosynthesis were upregulated in the spleen. These results not only explain the mechanism by which Tregs naturally recover but also indicate that changes in Tregs differ between the blood and spleen. Differentially-expressed proteins serving a role in these pathways provide insight into novel targets for sepsis detection and therapy. Further studies on a larger sample set are required to verify the results of the current study and to confirm the roles of individual differentially-expressed proteins.

\section{Acknowledgements}

The current study was performed in the Laboratory of Molecular Biology of the Second Hospital of Dalian Medical University and Dalian Institute of Chemical Physics, Chinese Academy of Sciences (Dalian, China) and was supported by the National Natural Science Foundation of China (Beijing, China, awarded to J.X., grant no. 81171791).

\section{References}

1. Singer M, Deutschman CS, Seymour CW, Shankar-Hari M, Annane D, Bauer M, Bellomo R, Bernard GR, Chiche JD, Coopersmith CM, et al: The third international consensus definitions for sepsis and septic shock (Sepsis-3). JAMA 315: 801-810, 2016.

2. Stoller J, Halpin L, Weis M, Aplin B, Qu W, Georgescu C and Nazzal M: Epidemiology of severe sepsis: 2008-2012. J Crit Care 31: 58-62, 2016.

3. Yadav Hand Cartin-Ceba R: Balance between Hyperinflammation and Immunosuppression in Sepsis. Semin Respir Crit Care Med 37: 42-50, 2016.

4. Hotchkiss RS, Monneret G and Payen D: Sepsis-induced immunosuppression: From cellular dysfunctions to immunotherapy. Nat Rev Immunol 13: 862-874, 2013.

5. Hotchkiss RS, Monneret G and Payen D: Immunosuppression in sepsis: A novel understanding of the disorder and a new therapeutic approach. Lancet Infect Dis 13: 260-268, 2013.

6. Attridge $\mathrm{K}$ and Walker LS: Homeostasis and function of regulatory T cells (Tregs). in vivo: Lessons from TCR-transgenic Tregs. Immunol Rev 259: 23-39, 2014.

7. Guo J and Zhou X: Regulatory T cells turn pathogenic. Cell Mol Immunol 12: 525-532, 2015.

8. Leavy O: Regulatory T Cells: Distinct role in tissue repair. Nat Rev Immunol 15: 596-597, 2015.

9. Cao C, Ma T, Chai YF and Shou ST: The role of regulatory $\mathrm{T}$ cells in immune dysfunction during sepsis. World J Emerg Med 6: 5-9, 2015.

10. Nascimento DC, Alves-Filho JC, Sônego F, Fukada SY, Pereira MS, Benjamim C, Zamboni DS, Silva JS and Cunha FQ: Role of regulatory $\mathrm{T}$ cells in long-term immune dysfunction associated with severe sepsis. Crit Care Med 38: 1718-1725, 2010.

11. Rudiger A, Dyson A, Felsmann K, Carré JE, Taylor V, Hughes S, Clatworthy I, Protti A, Pellerin D, Lemm J, et al: Early functional and transcriptomic changes in the myocardium predict outcome in a long-term rat model of sepsis. Clin Sci (Lond) 124: 391-401, 2013.

12. Brealey D, Karyampudi S, Jacques TS, Novelli M, Stidwill R, Taylor V, Smolenski RT and Singer M: Mitochondrial dysfunction in a long-term rodent model of sepsis and organ failure. Am J Physiol Regul Integr Comp Physiol 286: R491-R497, 2004.

13. Lilley E, Armstrong R, Clark N, Gray P, Hawkins P, Mason K, López-Salesansky N, Stark AK, Jackson SK, Thiemermann C and Nandi M: Refinement of animal models of sepsis and septic shock. Shock 43: 304-316, 2015.

14. Fink MP: Animal models of sepsis. Virulence 5: 143-153, 2014.
15. He X, Wei Z, Zhou E, Chen L, Kou J, Wang J and Yang Z: Baicalein attenuates inflammatory responses by suppressing TLR4 mediated NF- $\mathrm{NB}$ and MAPK signaling pathways in LPS-induced mastitis in mice. Int Immunopharmacol 28: 470-476, 2015.

16. Kim J, Yang HY and Jang YS: A G protein-associated ERK pathway is involved in LPS-induced proliferation and a PTK-associated p38 MAPK pathway is involved in LPS-induced differentiation in resting B cells. Mol Immunol 43: 1232-1242, 2006.

17. Gonzalo S, Grasa L, Arruebo MP, Plaza MÁ and Murillo MD: Extracellular signal-regulated kinase (ERK) is involved in LPS-induced disturbances in intestinal motility. Neurogastroenterol Motil 23: e80-e90, 2011.

18. Asati V, Mahapatra DK and Bharti SK: PI3K/Akt/mTOR and Ras/Raf/MEK/ERK signaling pathways inhibitors as anticancer agents: Structural and pharmacological perspectives. Eur J Med Chem 109: 314-341, 2016.

19. Yamashita M, Shinnakasu R, Asou H, Kimura M, Hasegawa A, Hashimoto K, Hatano N, Ogata M and Nakayama T: Ras-ERK MAPK cascade regulates GATA3 stability and Th2 differentiation through ubiquitin-proteasome pathway. J Biol Chem 280: 29409-29419, 2005.

20. Knight T and Irving JA: Ras/Raf/MEK/ERK pathway activation in childhood acute lymphoblastic leukemia and its therapeutic targeting. Front Oncol 4: 160, 2014.

21. Fischer TH, Gatling MN, McCormick F, Duffy CM and White GC II: Incorporation of Rap 1b into the platelet cytoskeleton is dependent on thrombin activation and extracellular calcium. J Biol Chem 269: 17257-17261, 1994.

22. Naci D and Aoudjit F: Alpha2beta1 integrin promotes T cell survival and migration through the concomitant activation of ERK/Mcl-1 and p38 MAPK pathways. Cell Signal 26: 2008-2015, 2014.

23. Gendron S, Couture J and Aoudjit F: Integrin alpha2beta1 inhibits Fas-mediated apoptosis in T lymphocytes by protein phosphatase 2A-dependent activation of the MAPK/ERK pathway. J Biol Chem 278: 48633-48643, 2003.

24. Iwamoto DV and Calderwood DA: Regulation of integrin-mediated adhesions. Curr Opin Cell Biol 36: 41-47, 2015.

25. Mattila PK, Batista FD and Treanor B: Dynamics of the actin cytoskeleton mediates receptor cross talk: An emerging concept in tuning receptor signaling. J Cell Biol 212: 267-280, 2016.

26. Hirata H, Chiam KH, Lim CT and Sokabe M: Actin flow and talin dynamics govern rigidity sensing in actin-integrin linkage through talin extension. J R Soc Interface 11: pii: 20140734, 2014.

27. Critchley DR and Gingras AR: Talin at a glance. J Cell Sci 121: 1345-1347, 2008

28. Priddle H, Hemmings L, Monkley S, Woods A, Patel B, Sutton D, Dunn GA, Zicha D and Critchley DR: Disruption of the talin gene compromises focal adhesion assembly in undifferentiated but not differentiated embryonic stem cells. J Cell Biol 142: 1121-1133, 1998

29. Zhao P, Ma W, Hu Z, Zang L, Tian Z and Zhang K: Filamin A (FLNA) modulates chemosensitivity to docetaxel in triple-negative breast cancer through the MAPK/ERK pathway. Tumour Biol 37: 5107-5115, 2016.

30. Shao QQ, Zhang TP, Zhao WJ, Liu ZW, You L, Zhou L, Guo JC and Zhao YP: Filamin A: Insights into its Exact Role in Cancers. Pathol Oncol Res 22: 245-252, 2016.

31. Truong T, Shams H and Mofrad MR: Mechanisms of integrin and filamin binding and their interplay with talin during early focal adhesion formation. Integr Biol (Camb) 7: 1285-1296, 2015.

32. De Franceschi $\mathrm{N}$ and Ivaska J: Integrin bondage: Filamin takes control. Nat Struct Mol Biol 22: 355-357, 2015.

33. Modarres HP and Mofradt MR: Filamin: A structural and functional biomolecule with important roles in cell biology, signaling and mechanics. Mol Cell Biomech 11: 39-65, 2014.

34. Winkler GS: The mammalian anti-proliferative BTG/Tob protein family. J Cell Physiol 222: 66-72, 2010.

35. Berthet C, Guéhenneux F, Revol V, Samarut C, Lukaszewicz A, Dehay C, Dumontet C, Magaud JP and Rouault JP: Interaction of PRMT1 with BTG/TOB proteins in cell signalling: Molecular analysis and functional aspects. Genes Cells 7: 29-39, 2002.

36. Liu C, Tao T, Xu B, Lu K, Zhang L, Jiang L, Chen S, Liu D, Zhang X, Cao N and Chen M: BTG1 potentiates apoptosis and suppresses proliferation in renal cell carcinoma by interacting with PRMT1. Oncol Lett 10: 619-624, 2015. 
37. Voo KS, Foglietta M,Percivalle E, Chu F, Nattamai D, Harline M, Lee ST, Bover L, Lin HY, Baladandayuthapani V, et al: Selective targeting of Toll-like receptors and OX40 inhibit regulatory $\mathrm{T}$-cell function in follicular lymphoma. Int J Cancer 135 : 2834-2846, 2014.

38. Vu MD, Xiao X, Gao W, Degauque N, Chen M, Kroemer A Killeen N, Ishii N and Li XC: OX40 costimulation turns off Foxp3 ${ }^{+}$Tregs. Blood 110: 2501-2510, 2007.

39. Hawse WF, Gloor BE, Ayres CM, Kho K, Nuter E and Baker BM: Peptide modulation of class I major histocompatibility complex protein molecular flexibility and the implications for immune recognition. J Biol Chem 288: 24372-24381, 2013.

40. Rudolph MG, Stanfield RL and Wilson IA: How TCRs bind MHCs, peptides and coreceptors. Annu Rev Immunol 24 419-466, 2006

41. Liu B, Zheng Y, Yin F, Yu J, Silverman N and Pan D: Toll receptor-mediated hippo signaling controls innate immunity in drosophila. Cell 164: 406-419, 2016.

42. Ye S and Eisinger-Mathason TS: Targeting the Hippo pathway: Clinical implications and therapeutics. Pharmacol Res 103: 270-278, 2016

43. Mo JS, Park HW and Guan KL: The Hippo signaling pathway in stem cell biology and cancer. EMBO Rep 15: 642-656, 2014

44. Pan D: The hippo signaling pathway in development and cancer. Dev Cell 19: 491-505, 2010.

45. Yu FX and Guan KL: The Hippo pathway: Regulators and regulations. Genes Dev 27: 355-371, 2013.

46. Tumaneng K, Schlegelmilch K, Russell RC, Yimlamai D, Basnet H, Mahadevan N, Fitamant J, Bardeesy N, Camargo FD and Guan KL: YAP mediates crosstalk between the Hippo and PI(3)K-TOR pathways by suppressing PTEN via miR-29. Nat Cell Biol 14: 1322-1329, 2012.

47. Liko D and Hall MN: mTOR in health and in sickness. J Mol Med (Berl) 93: 1061-1073, 2015.

48. Sandilands E, Schoenherr C and Frame MC: p70S6K is regulated by focal adhesion kinase and is required for Src-selective autophagy. Cell Signal 27: 1816-1823, 2015.

49. Tabe Y, Jin L, Konopleva M, Shikami M, Kimura S, Andreeff M, Raffeld $M$ and Miida T: Class IA PI3K inhibition inhibits cell growth and proliferation in mantlecell lymphoma. Acta Haematol 131: 59-69, 2014
50. Vivanco I and Sawyers CL: The phosphatidylinositol 3-kinase AKT pathway in human cancer. Nat Rev Cancer 2: 489-501, 2002.

51. Ribeiro PS, Josué F, Wepf A, Wehr MC, Rinner O, Kelly G, Tapon $\mathrm{N}$ and Gstaiger M: Combined functional genomic and proteomic approaches identify a PP2A complex as a negative regulator of Hippo signaling. Mol Cell 39: 521-534, 2010.

52. Zeng Q, Zhang H, Qin J, Xu Z, Gui L, Liu B, Liu C, Xu C, Liu W, Zhang S, et al: Rapamycin inhibits BAFF-stimulated cell proliferation and survival by suppressing mTOR-mediated PP2A-Erk1/2 signaling pathway in normal and neoplastic B-lymphoid cells. Cell Mol Life Sci 72: 4867-4884, 2015.

53. Li Z, Tang J and Guo F: Identification of 14-3-3 proteins phosphopeptide-binding specificity using an affinity-based computational approach. PLoS One 11: e147467, 2016.

54. Bikle D: Nonclassic actions of vitamin D. J Clin Endocrinol Metab 94: 26-34, 2009.

55. Verstuyf A, Carmeliet G, Bouillon R and Mathieu C: Vitamin D: A pleiotropic hormone. Kidney Int 78: 140-145, 2010.

56. Baeke F, Takiishi T, Korf H, Gysemans C and Mathieu C: Vitamin D: Modulator of the immune system. Curr Opin Pharmacol 10: 482-496, 2010

57. Cheng JB, Levine MA, Bell NH, Mangelsdorf DJ and Russell DW: Genetic evidence that the human CYP2R1 enzyme is a key vitamin D 25-hydroxylase. Proc Natl Acad Sci USA 101: 7711-7715, 2004

58. Zawaira A, Gallotta M, Beeton-Kempen N, Coulson L, Marais P, Kuttel $\mathrm{M}$ and Blackburn J: Exhaustive computational search of ionic-charge clusters that mediate interactions between mammalian cytochrome P450 (CYP) and P450-oxidoreductase (POR) proteins. Comput Biol Chem 34: 42-52, 2010.

59. Gocek E, Marchwicka A, Bujko K and Marcinkowska E: NADPH-cytochrome $\mathrm{P} 450$ reductase is regulated by all-trans retinoic acid and by 1,25-dihydroxyvitamin D3 in human acute myeloid leukemia cells. PLoS One 9: e91752, 2014. 